\title{
Microfluidique discrète et biotechnologie
}

\author{
Yves Fouillet ${ }^{\mathrm{a}, *}$, Jean-Luc Achard ${ }^{\mathrm{b}}$ \\ a Laboratoire d'electronique et de technologie de l'information (LETI), CEA/LETI/DSIS/MSB - Grenoble, LETI, 17, avenue des Martyrs, \\ 38054 Grenoble cedex 9, France \\ ${ }^{\mathrm{b}}$ Laboratoire des ecoulements géophysiques et industriels (LEGI), INPG/CNRS/UJF-Grenoble, LEGI, 1021, rue de la Piscine, \\ 38400 St Martin d'Hères, France
}

La conception des microsystèmes d'analyse pour les biotechnologies, s'envisage suivant deux écoles de pensée : la microfluidique continue, plus ancienne, est fondée sur des écoulements dans les canaux ; la microfluidique discrète, manipule des gouttes. Les avantages de cette dernière, qui s'appuie sur l'ElectroHydroDynamique et l'électromouillage, sont soulignés, avec en particulier une capacité plus grande d'intégration dans les microsystèmes des fonctions fluidiques élémentaires. Deux familles de configuration planaires sont détaillées : ouvertes et confinées. Des réalisations en sont présentées et notamment un système utilisant un réseau de microcaténaires pour injecter, déplacer, fusionner, mélanger des gouttes etc.

Mots-clés : Gouttes ; Micofluidique Discrète ; Electromouillage ; ElectroHydroDynamique ; Labopuce

Digital microfluidic and biotechnology. Designing Microsystems for biotechnology analysis may be tackled according to two schools of thought: the older, continuous microfluidics, is based on of flows in channels, and digital microfluidics, which is based on droplet handling. The advantages of the latter, which is based on ElectroHydroDynamics and Electrowetting, are emphasized, with a particular focus on its enhanced capacity for integration of elementary fluidic functions into the microsystems. Two planar families of configuration are detailed, namely the opened and the confined. The microsystems achieved are then presented, together with the network of microcatenaries, used for injecting, moving, merging, mixing drops etc.

Keywords: Droplets; Digital microfluidic; Electrowetting; ElectroHydroDynamique; Lab On Chip

\section{Introduction}

Dans les années 1970, on commence à noter des travaux précurseurs [1] proposant d'utiliser les microtechnologies déjà développées dans le domaine des MEMS (Micro-Electro-Mechanical Systems), pour intégrer des protocoles chimiques. Mais la notion de laboratoire sur puce (labopuce dans la suite de l'article et en Anglais, Lab On Chip, ou encore Micro Total Analysis System ne fut véritablement introduite par Manz qu'à la fin des années 1980 [2]. Idéalement, un labopuce peut être décrit comme un système d'analyse chimique ou biologique complètement miniaturisé et intégré dans un seul composant. Depuis les

\footnotetext{
* Auteur de correspondance.

Adresses e-mail : yfouillet@ cea.fr (Y. Fouillet), Jean-Luc.Achard@hmg.inpg.fr (J.-L. Achard).
} 
années 1990, on assiste à un accroissement quasi exponentiel de publications et d'études dans ce domaine. De la génomique, en passant par la protéomique jusqu'à l'étude de la cellule, de nombreux domaines de la biologie sont concernés par la mise en place de microsystèmes d'analyse fondés sur des technologies et des principes physiques très variés. Se profile donc la constitution d'une formidable boite à outils et le rêve serait, en transposant ici la loi de Moore [3] qui scande les succès de la microélectronique, d'intégrer sans cesse un plus grand nombre d'analyses sur une même surface de puce; un aboutissement pourrait être la réalisation de véritables processeurs fluidiques [4].

Il faudra cependant attendre le début des années 2000 pour voir apparaître le premier système commercial (Caliper Bioanalyzer). Malgré quelques succès économiques qui ont suivi, force est de constater que le nombre de réussites reste encore négligeable eu égard aux nombreuses tentatives. Il est en effet reconnu qu'il existe encore un certain nombre de blocages. En microfluidique, une des approches les plus répandues pour déplacer les fluides d'un point à un autre d'un labopuce est de les pomper à travers un réseau de canaux micro-usinés; par soucis de concision, cette approche sera appelée par la suite «microfluidique continue», dénomination qui n'exclut pas des écoulements diphasiques comportant des bouchons fluides au sein des canaux que nous définirons dans la Section 2. Grâce aux microtechnologies, une grande variété de micro-pompes (et inversement de vannes) ont été conçues, fondées par exemple sur la piézoélectricité, l'électrostatique, la thermique, le magnétisme, l'électrocinétique... [5]. De surcroît, il ne s'agit pas seulement de déplacer (et inversement de bloquer) les fluides, mais de réaliser toute une série d'opérations sur ces fluides requises par le protocole : injection, division ou réunion de flux, mélange de fluide, dosage de réactif, extraction ou identification de substances, récupération etc. Cette problématique de processus industriel s'inscrit dans une sorte de génie des procédés microfluidiques qui possède ses propres spécificités, comme celle d'inclure correctement l'interfaçage du labopuce avec le monde macroscopique de l'opérateur. Néanmoins, lors de mise en œuvre d'un microsystème d'analyse sur puce, cette problématique se trouve très souvent mise en arrière plan et reléguée en fin d'étude. Un des objectifs de cet article est d'en montrer toute la complexité.

Cette problématique va être détaillée dans le seul cadre d'une autre approche plus récente et donc moins répandue, appelée par la suite «microfluidique discrète» [6]. Cette approche consiste à réaliser les protocoles biologiques au sein de gouttes de fluides biologiques ou de réactifs que l'on souhaite manipuler (c'est-à-dire, injecter, prélever, déplacer, fusionner, morceller, mélanger, récupèrer etc) à volonté. Ces opérations doivent être assurées indépendamment sur chaque goutte présente dans les composants qui peuvent être alors assimilées à des micro-robots. Les gouttes, véritables microréacteurs constituées par une solution aqueuse dont la composition est imposée par les biologistes, sont immergées dans un fluide continu. La première fonction de ce fluide est de former une barrière physique pour confiner les volumes de réaction; l'air peut ainsi être choisi. Afin de supprimer tout phénomène d'évaporation, un liquide non miscible comme l'huile peut être préféré. Ainsi, sur le plan électrique, le système en jeu combine des gouttes plutôt conductrices dans un fluide plutôt diélectrique confiné par un ou des plans rigides à champ de potentiel commandé. A certains égards, la première fonction d'une goutte est d'imiter le rôle de l'empedorf du biologiste ; on peut même comparer un microsystème à microfluidique discrète à une chaîne robotisé de gestion de plaque à puits miniaturisée.

Sur cette branche particulière de la microfluidique dédiée aux labopuces, de nombreux travaux ont déjà été publiés. Sur la seule opération de déplacement des gouttes, les principes proposées sont multiples, comme les ondes acoustiques de surface, la thermocapillarité [7], la microstructuration de surface asymétrique [8] etc. Notre objectif ici est nullement d'établir une sorte d'inventaire exhaustif de ces méthodes, qui serait à la fois impossible à réaliser, tant les innovations foisonnent, et également très fastidieux. Il nous paraît, et il y a certainement là une part d'arbitraire, que la discipline scientifique dont est issu le plus grand nombre de solutions techniques fiables et commodes à piloter dans le domaine qui nous intéresse, est l'ElectroHydroDynamique [9], notée EHD dans la suite. Les forces qui peuvent être mises à profit sont les forces coulombiennes et diélectrophorétiques, les forces d'entraînement dues aux injections d'ions, etc. Un bref rappel sur la définition de ces forces sera présentée dans la Section 3 ; on y parlera également de l'électromouillage, phénomène extrêmement important dans cette microfluidique, qui donne également lieu à des forces de type électroquasistatique. Quelques opérations classiques et réussies, réalisées en mettant en œuvre ces forces, seront présentées dans les Sections 4 et 5. Mais auparavant il est important de distinguer les deux systèmes d'analyse principaux que l'on rencontre dans les biotechnologies. Malgré des différences évidentes, nous allons voir qu'ils ressortissent bien à une problématique commune à l'ensemble des microsystèmes dédiés aux biotechnologies.

\section{Problématique fluidique des microsystèmes dédiés aux biotechnologies}

Pour les systèmes d'analyses sur le terrain (Point-Of-Care) [10], l'objectif est de réaliser quelques analyses sur un même échantillon à l'aide de composants qui sont généralement jetable après une seule analyse. Par conséquent, il est impératif de trouver des solutions peu coûteuses (i) pour fabriquer en masse de tels composants consommables intégrant plusieurs fonctions, (ii) pour automatiser la connection automate-composant, (iii) gérer en aveugle la microfluidique des différentes fonctions élémentaires relatives aux étapes du protocoles : dosage, déplacement, mélange, incubation etc. 

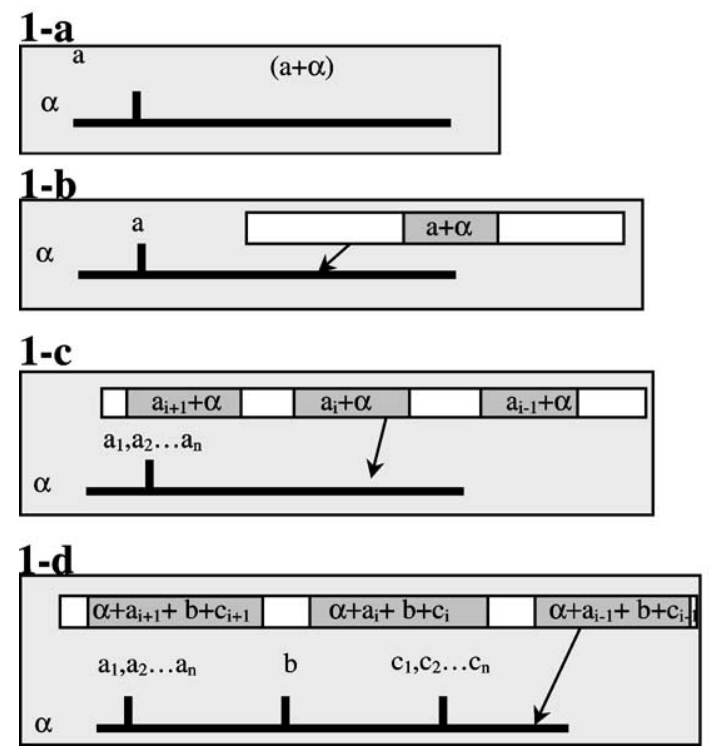

1-e
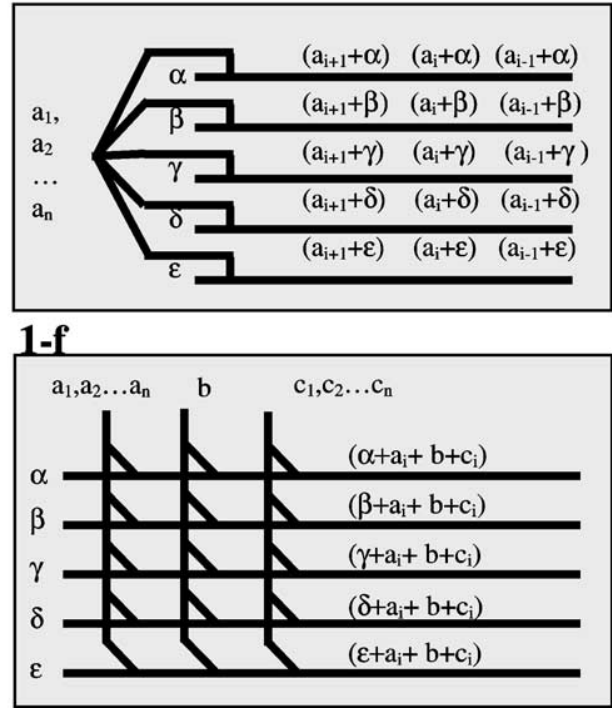

Fig. 1. Exemples de schémas fluidiques : un liquide à analyser est indiqué par une lettre grecque tandis une classe de réactifs, attaché à un port particulier d'émission, est indiqué par une lettre latine. Un représentant dans une classe de réactifs, caractérisé ici par son ordre d'injection, est signalé par un indice inférieur.

Il existe une deuxième famille de solution où le microsystème doit réaliser un très grand nombre d'analyses. C'est le domaine du criblage, du séquençage ou du génotypage. Ici les contraintes sont toujours d'abaisser le coût d'une campagne de test mais aussi d'augmenter le débit d'analyse. Il faut donc réduire les volumes de réactifs, et assigner aux composants de gérer simultanément un très grand nombre de réaction sur un même support.

Commune aux deux familles, une hiérarchie de schémas fonctionnels, dont la complexité croît suivant la nature du protocole à intégrer, est présentée dans la Fig. 1. En se situant dans le cadre de la microfluidique continue, montrons dans chaque cas les difficultés de mise en œuvre de ces schémas avant de considérer les solutions qu'offre, pour les mêmes cas, la microfluidique discrète.

Le premier protocole (Fig. 1-a) consiste à injecter en continu deux liquides $(\alpha)$ et $(a)$ sur un même canal; $(\alpha)$ peut être une préparation obtenue à partir d'un prélèvement biologique tandis que $(a)$ peut être un révélateur pour détecter une substance donnée dans $(\alpha)$. On souhaite un mélangeage rapide de ces liquides. Le nombre de Reynolds $R e=U d / \mu$ (ou $U$ est une échelle de vitesse, $d$, une échelle de longueur, et $v$, la viscosité cinématique) étant très petit, toute instabilité hydrodynamique se voit inhibée et la diffusion Brownienne se retrouve seule pour assurer le mélange des espèces. On est donc conduit à assister la diffusion en suscitant en continu des mouvements à petites échelles du fluide [11].

Il est généralement impératif de réduire les volumes de réactifs utilisés, voire de liquide à analyser. Pour cela, il faut injecter (a) suivant un laps de temps limité plutôt qu'en continu. Du point de vue du canal dans lequel $(\alpha)$ est injecté, deux options différentes se présentent : la voie monophasique et la voie diphasique. Dans la première option, où le fluide à analyser est continûment injecté (un seul échantillon), on créé ainsi une zone réactionnelle qui tend à s'étaler au cours du temps au sein de ce fluide. Dans la deuxième option, où le fluide à analyser est concentré en un bouchon, limité en amont et en aval par un fluide inerte, on réduit de plus $(\alpha)$. Dans la Fig. 1-b, le segment grisé représente aussi bien une zone réactionnelle, dont les limites diffuses sont floues, qu'un bouchon, dont les limites entre deux phases sont nettes.

La Fig. 1-c représente le cas, plus complexe, où plusieurs analyses différentes, mais relatives à une même classe de réactifs, doivent être effectuées sur un même liquide $(\alpha)$. Par exemple $(\alpha)$ est une préparation contenant de l'ADN génomique dont on désire analyser $n$ gènes et $a_{i}(i$ varie de 1 à $n)$ représente le réactif particulier détectant le gène indicé par $i$, numéro correspondant suivant notre convention à l'ordre $i$ de son injection. A partir d'un automate, permettant de basculer de l'injection $i$ à l'injection $i+1$, il faut donc réaliser $n$ mélanges réactionnels au sein d'un échantillon de $(\alpha)$. Ici encore se présente la double option, monophasique ou diphasique. Dans la première option, on crée $n$ zones réactionnelles $\left[\alpha+a_{1}\right]$, $\left[\alpha+a_{2}\right], \ldots,\left[\alpha+a_{i}\right], \ldots,\left[\alpha+a_{n}\right]$ dans le liquide $(\alpha)$. Ces zones sont maintenues indépendantes par un espacement suffisant le long du canal. En effet, il faut se prémunir du risque de connexion axiale entre zones provoquée par la diffusion Brownienne, (favorable par ailleurs au mélange dans chaque zone), mais également par la diffusion de Taylor [12]. Il en résulte une diminution de la fréquence d'injection des réactifs à un port donné ; et donc du débit d'analyse. Un profil de vitesse plat obtenu par un pompage électoosmotique est plus adapté mais reste plus complexe à maîtriser. Dans la seconde option, on localise chaque 
réaction au sein de bouchons successifs entraînés dans un liquide connexe et totalement mouillable ; l'échantillon initial se voit alors fragmenté en autant d'éléments que de bouchons. Une grande régularité spatio-temporelle de l'écoulement diphasique résultant, difficile à obtenir quand le nombre capillaire $C a=U \eta / \sigma$ (où $\sigma$ est la tension superficielle et $\eta$ la viscosité dynamique) crôt, est exigée par les différentes interventions. Une seconde variante peut être d'éliminer les films liquides autour des bouchons en utilisant un liquide inerte mouillant partiellement la paroi; les bouchons ne sont alors délimités que par des ménisques. La relation débit-pression devient chaotique et une méthode d'injection à débit imposé (via par exemple un pousseseringue) se révèle in fine indispensable. La distinction entre ces deux variantes est en fait de peu d'intérêt dans la réalité industrielle. La présence de tensio-actif hydrophobes, les formes changeantes des canaux, leur état de surface variable, etc. sont autant de facteurs aléatoires qui empêchent une maîtrise des conditions de mouillabilité en régime dynamique.

La Fig. 1-d, représente un cas, encore plus délicat, où plusieurs analyses différentes et relatives à plusieurs classes de réactifs, doivent être effectuées sur un même liquide $(\alpha)$. Toutes les difficultés décrites précédemment subsistent tout en étant amplifiées par la plus grande complexité du protocole à plusieurs étapes $\left(a_{i}, b_{j}, c_{k}\right)$; la difficulté majeure est de contrôler parfaitement le déplacement des bouchons dans les canaux par rapport aux différentes séquences d'injections relatives à chaque port. On peut parler de multiplexage fluidique puisque sur une voie donnée, chaque bouchon peut contenir une ou plusieurs informations après réaction. Un niveau supplémentaire de complexité est atteint quand il faut mettre en parallèle les protocoles sur plusieurs canaux le long desquels il faut multiplexer des réactions. Les Figs. 1-e et 1-f correspondent aux parallèlisations des schémas 1-c et 1-d respectivement. Dans une application relative au deuxième cas [13], nous avons estimé une précision de débit à $\pm 1 \%$.

On le voit : l'enjeu en microfluidique continue est non seulement de concevoir des innovations ingénieuses relatives à des opérations élémentaires (mélange, injection, séparation etc.) mais encore de les intégrer facilement dans un système complet.

La plupart des difficultés que nous venons d'évoquer, limitant l'intérêt de la miniaturisation, disparaissent en microfluidique discrète. Il devient en effet possible d'agir individuellement sur chaque goutte, sans perturber les gouttes voisines. La maîtrise acquise pour déplacer une goutte seule, peut être transposée sans encombre pour le déplacement d'un grand nombre de gouttes, et il en est de même pour toutes les fonction de bases que nous allons détailler. De plus l'aspect planaire du microsystème autorise un déplacement suivant deux directions; par cette propriété dont est naturellement privée la microfluidique continue essentiellement linéique, la microfluidique discrète se trouve bien adaptée aux problématiques de multiplexage et de synchronisation des réactions.

A titre d'exemple, la Fig. 2 représente une des configurations envisageables pour transposer le schéma fluidique de la Fig. 1-f. Les différents liquides réactifs sont stockés dans de petits réservoirs à la partie supérieure. Des gouttelettes, issues de ces réservoirs, sont distribuées vers la partie inférieure, en colonnes puis en lignes où les différentes réactions s'effectuent séquentiellement et parallèlement. Ainsi, l'utilisateur n'a plus qu'a remplir les différents réservoirs dans la zone de stockage en début d'analyse, et le composant assure la gestion fluidique interne. Notons que le volume de remplissage varie entre le $\mu$ l et le $\mathrm{ml}$ alors que les volumes des gouttes manipulés par le composant varient entre le nl et le $\mu$ l. Cela résout en grande partie les problèmes de volume mort (ou volume perdu) et simplifie l'interfaçage du composant avec le monde extérieur. Que ce soit pour

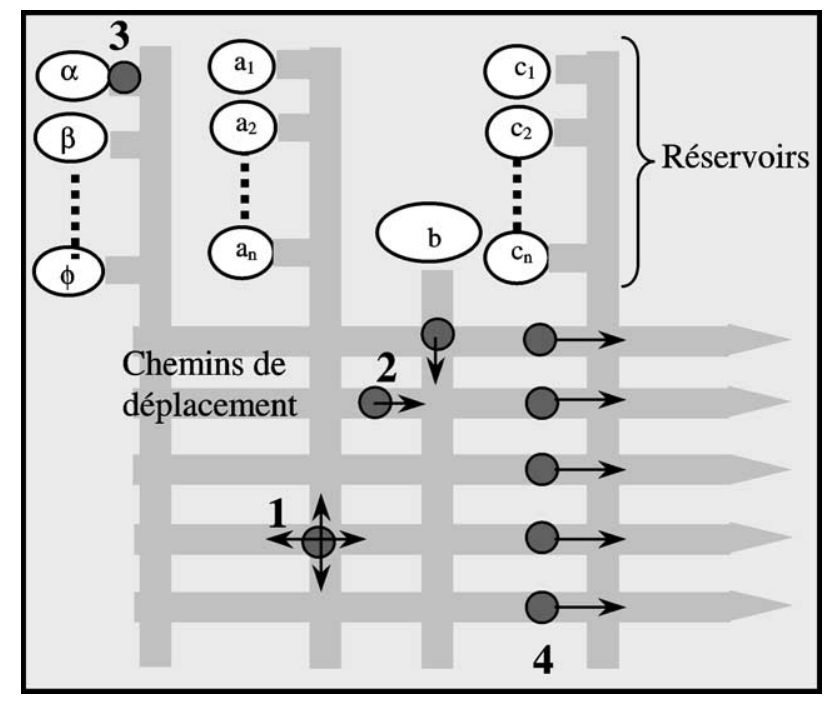

Fig. 2. Schéma d'un composant à microfluidique discrète pouvant réaliser un protocole fluidique équivalent au schéma 1f. Quelques fonctions de base sont représentées : déplacement $X-Y(1)$; mélange (2); séparation ou formation d'une goutte à partir d'un réservoir (3); synchronisation de la commande de déplacement (4). 
un composant dédié aux diagnostics, ou pour une application à haut débit d'analyse, le remplissage s'avère aisé : utilisation manuelle d'une pipette dans le premier cas, robot de dispense classique opérant sur un plus grand nombre de réservoirs dans le second cas.

En conclusion, pour que le composant assure la gestion interne des fluides, une maîtrise précise d'un nombre limité de fonctions de base est requise : le déplacement de gouttes suivant deux directions perpendiculaires, la fusion de deux gouttes ainsi que le mélange de leur constituants, le morcellement (dit aussi division ou encore fragmentation) de gouttes et enfin la formation des gouttelettes à partir des zones des réservoirs de stockage. Bien évidemment d'autres fonctions, qui ne relèvent pas strictement de la fluidique (cyclage thermique, lecture des résultats de réaction,...), sont à prévoir et ne seront pas décrites ici.

\section{Bref survol des phénomènes physiques mis en jeu}

L'EHD est fondée sur le couplage des équations de l'hydrodynamique et des équations de Maxwell réduites aux équations de l'électroquasistatique. Les conditions opératoires qui conduisent aux équations de l'électroquasistatique sont discutées dans [9]. Bornons nous ici à observer que ces conditions sont très souvent vérifiées dans les fluides diélectriques où les courants électriques sont faibles et les effets magnétiques négligeables; dans les systèmes qui nous occupent, la phase continue autour des gouttes est toujours constituée de ces fluides qui par là limitent les courants entre électrodes. Les trois types d'équations qui gèrent les champs électriques et les distributions de charges sont :

Les lois réduites de Faraday, $\nabla \times E=0$, dans chaque phase, qui permettent d'introduire le potentiel $V$ pour décrire le champ électrique $E=-\nabla V$. A l'interface, cette loi conduit à rendre les potentiels continus, $V_{\mathrm{e}}=V_{\mathrm{i}}$, où les indices inférieurs indiquent les fluides externes et internes respectivement.

Les lois de Gauss, $\nabla \cdot \varepsilon \mathbf{E}=q$, dans chaque phase de permittivité $\varepsilon$, avec $q$ représentant la densité volumique de charge libre. L'adaptation de cette loi à l'interface permet de connaître la valeur des charges libres $q_{\mathrm{s}}$ qui s'y sont accumulées (effet de Maxwell-Wagner), comme différence des composantes normales des vecteurs déplacement des deux milieux à l'interface $\llbracket \mathbf{n} \cdot \varepsilon \mathbf{E} \rrbracket=q_{\mathrm{s}}$. Dans cette condition de saut à l'interface, la notation $\llbracket \mathbf{n} \cdot \mathbf{F} \rrbracket=\sum_{k=\mathrm{i}, \mathrm{e}} \mathbf{n}_{k} \cdot \mathbf{F}_{k}$ est utilisée pour représenter les effets des milieux adjacents ; le vecteur unitaire normal, extérieur à la phase $k, y$ est noté $\mathbf{n}_{k}$. La permittivité dans l'eau est bien supérieure à la permittivité du fluide diélectrique. Le vecteur déplacement est donc très faible dans les gouttes, et la valeur des charges superficielles dépend des composantes normales à l'interface du seul vecteur déplacement électrique dans le fluide externe.

Les bilans de charges, $\partial q / \partial t+\nabla \cdot(q \mathbf{u}+\mathbf{J})=0$, dans chaque phase, avec $\mathbf{J}$ la densité de courant qui peut prendre plusieurs formes. Dans les cas qui nous intéressent avec peu d'impuretés, le milieu est souvent homogène et la loi d'Ohm peut-être adoptée $\mathbf{J}=\sigma \mathbf{E}$. On peut aussi vouloir pomper le fluide diélectrique et injecter pour cela une espèce d'ions via une électrode émettrice en pointe ou via un système de peignes d'électrodes émettrices et collectrices interdigitées [14]. Dans ce cas $\mathbf{J}=q \mu \mathbf{E}+D \nabla q$ où $\mu$ et $D$ sont la mobilité et le coefficient de diffusion respectivement, de l'espèce d'ion considérée. Aux interfaces les densités de courant se raccordent par un bilan de charges qui s'écrit $\partial q_{\mathrm{s}} / \partial t+q_{\mathrm{s}}\left(\mathbf{v} \cdot \mathbf{n}_{\mathrm{i}}\right) \nabla_{\mathrm{s}} \cdot \mathbf{n}_{\mathrm{i}}+\left[\left[\mathbf{n} \cdot \mathbf{J} \rrbracket=\mathbf{0}\right.\right.$ où $\left(\mathbf{v} \cdot \mathbf{n}_{\mathrm{i}}\right)$ est la vitesse de déplacement de l'interface et où $\nabla_{\mathrm{S}}$ est le gradient surfacique [15]. La conductivité dans l'eau est bien supérieure à la conductivité du fluide diélectrique. Bien que la goutte soit le siège d'un courant, celui-ci nécessite donc une variation de potentiel très faible au sein des gouttes par rapport à la variation dans le fluide diélectrique. Ce point apparaît clairement dans le bilan des charges précédent libres à l'interface. En dehors de tout contact avec des électrodes, les gouttes sont donc quasi isopotentielles, et en particulier, le champ électrique tangent à l'interface est quasi nul. Par conséquent, celles des lignes de courant allant d'une électrode à une autre qui passent par les gouttes entrent dans celles-ci normalement et en émergent de même.

Comment les équations de l'hydrodynamique sont elles couplées à ces dernières équations de l'électroquasistatique ? Il existe de façon générale trois forces volumiques de nature électrique dans l'équation de quantité de mouvement : la force de Coulomb, $q \mathbf{E}$, la force diélectrique et la force électrorésistive. La première force est importante en particulier lors d'injection d'ions. La deuxième force résulte des variations de $\varepsilon$ dans l'espace. La troisième fait intervenir la dépendance de $\varepsilon$ en fonction de la masse volumique. Ces deux dernières sont négligeables pour les cas qui nous intéressent. Il reste donc qu'en général le couplage qui vient d'être évoqué se situe essentiellement aux interfaces grâce aux équations de bilan de quantité de mouvement, normal et tangentiel, que nous allons rappeler :

$$
\begin{aligned}
& \llbracket \mathbf{n} \cdot \overline{\overline{\mathbf{T}}} \rrbracket \cdot \mathbf{n}_{\mathrm{i}}+\sigma \nabla_{\mathrm{s}} \cdot \mathbf{n}_{\mathrm{i}}=\llbracket \varepsilon\left[\frac{1}{2} E^{2}-(\mathbf{n} \cdot \mathbf{E})^{2}\right] \mathbf{n} \cdot \overline{\overline{\mathbf{I}}} \rrbracket \cdot \mathbf{n}_{\mathrm{i}}, \\
& 2 \llbracket \mathbf{n} \cdot \eta \overline{\overline{\mathbf{D}}} \rrbracket \cdot \mathbf{a}_{\alpha}-\left(\nabla_{\mathrm{s}} \sigma\right) \cdot \mathbf{a}_{\alpha}=-q_{s} \mathbf{E} \cdot \mathbf{a}_{\alpha}, \quad \alpha=1,2,
\end{aligned}
$$

équations où les contraintes électriques ont été rassemblées à droite et les contraintes hydrodynamiques à gauche. Dans ces équations : 
$\underline{p}$ est la pression,

$\overline{\overline{\mathbf{D}}}$ est la partie symétrique du gradient de la vitesse $\mathbf{u}$,

$\overline{\overline{\mathbf{T}}}=-p \overline{\overline{\mathbf{I}}}+2 \eta \overline{\overline{\mathbf{D}}}$ est le tenseur des contraintes hydrodynamiques,

$\nabla_{\mathrm{S}} \cdot \mathbf{n}_{\mathrm{i}}$ qui est la divergence du vecteur unitaire pointant à l'extérieur d'une goutte, permet d'exprimer la courbure moyenne dans la force de Laplace,

$\nabla_{\mathrm{S}} \sigma$ est le gradient surfacique de $\sigma$ et représente donc la force de Marangoni liée à toute cause (thermique ou chimique) faisant varier $\sigma$,

$\mathbf{a}_{\alpha}$ représente un des deux vecteurs tangents à l'interface.

L'ensemble des équations que nous venons brièvement d'évoquer permet de formuler des problèmes aux limites cohérents, schématisant les parties des composants que l'on veut concevoir où optimiser. Leur résolution, analytique mais le plus souvent numérique, offre un support à la réflexion que nous estimons essentiel pour la conduite de l'inévitable approche expérimentale. Or l'approche habituelle de l'ingénieur est plutôt de s'appuyer sur la résolution, disponibles dans la littérature, de problèmes simplifiés types auxquels le système qui est étudié peut être plus ou moins assimilé. Dans le domaine de l'EHD, on trouve par exemple les expressions des forces diélectrophorétiques agissant sur une sphère plongée dans un fluide infini soumis à un gradient de champ électrique ou encore de la force d'attraction ou de répulsion entre deux sphères plongées dans un fluide infini soumis à un champ électrique uniforme [16]. Les expressions de ces forces mettent en évidence des moments dipolaires qui sont des fonctions des conductivités et des permittivités des deux phases intérieures et extérieures, modulées par la fréquence du courant utilisé quand celui ci-est alternatif. Les restrictions très fortes au sein desquelles ces résultats ont été établis ne doivent pas être oubliées. Il apparaît alors que ces résultats ont un intérêt bien minces vis à vis des phénomènes physiques complexes auxquels nous sommes confrontés ; évoquons ainsi trois sources de complexité. D’abord les systèmes étudiés sont confinés et les frontières limitant le fluide diélectrique autour d'une goutte sont variées : autres gouttes, capillaires, électrodes planes etc. Ensuite les opérations étudiées comme la coalescence ou le morcellement sont transitoires; elles correspondent enfin à des problèmes aux frontières libres difficiles : les gouttes ne demeurent plus sphériques. En conclusion, il n'est généralement pas possible d'éluder, l'étude approfondie de problèmes physiques nouveaux poursuivant ainsi, dans un contexte nouveau, l'oeuvre de pionnier entreprise par Taylor [17] dans ce domaine.

L'électromouillage qui n'est traditionnellement pas inclus dans l'EHD permet également de coupler hydrodynamique et champs électriques en diminuant l'angle de contact d'une goutte posée sur un support solide par application d'une différence de potentiel. Cette dernière est appliquée entre le support lui-même et une électrode plongée dans la goutte. Dans le cas où le liquide est en contact direct avec l'électrode-support, on parle d'électromouillage (EW en anglais). Si la surface de contact est isolante et que l'électrode est enterrée sous l'isolant d'épaisseur e et de constante diélectrique $\varepsilon_{O} \varepsilon$, on parle d'électromouillage sur diélectrique (EWOD en anglais). Cette dernière variante est la plus connue et semble être la plus adaptée à nos objectifs. Le principe a été initialement étudiée par Berge [18]; à partir de la loi de Young, on obtient la formule de Lippman-Berge qui donne l'angle de contact en fonction du potentiel appliqué :

$$
\cos \theta(V)=\cos \theta_{o}+\frac{1}{2} \frac{C V^{2}}{\gamma_{L G}},
$$

où $\gamma_{L G}$ est la tension de surface gaz-liquide, $C=\varepsilon_{0} \varepsilon / e$ est la capacité de contact par unité de surface que constitue l'isolant, $\theta_{0}$ est l'angle de contact en l'absence de potentiel. La mise en œuvre de cette variante repose essentiellement sur la qualité de la couche isolante qui doit avoir un C élevé, une tension de claquage faible, une hydrophobie importante et une faible hystérésis de mouillage afin d'amplifier le phénomène [19]. D'après l'Éq. (3), il semble possible d'atteindre un mouillage total; en réalité, à partir d'une certaine valeur de la tension, il existe une saturation, qui peut être corrélée à des phénomènes d'éjection de gouttelettes satellites [20] ou de ionisation.

Une application de l'électromouillage est de permettre le déplacement d'une goutte ; celle-ci doit être placée sur un pavage d'électrode, chacune de taille inférieure à la zone mouillée. Si l'on actionne de façon asymétrique les électrodes en contact avec la goutte, on peut créer des différences d'angle de contact, promotrices d'un glissement en masse de la goutte.

Une interprétation des phénomènes par la seule prise en compte de (3) est insuffisante : la diélecrophorèse doit être aussi considérée. Il convient donc, encore ici, de replacer ce phénomène dans le cadre de l'EHD pour décrire la variété des situations, dont certaines sont dynamiques, auxquelles la microfluidique discrète est confrontée [21].

Passons précisément à la description de quelques unes de ces situations. Trois familles de configurations peuvent être distinguées, selon que la goutte est en lévitation complète, qu'elle repose sur un plan, ou encore qu'elle est confinée entre deux plans. Ici seuls les deux derniers cas seront abordés 


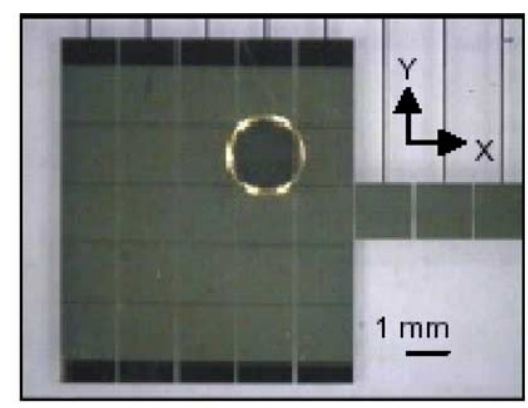

(a) Droplet is moving on a $5 \times 5$ grid.

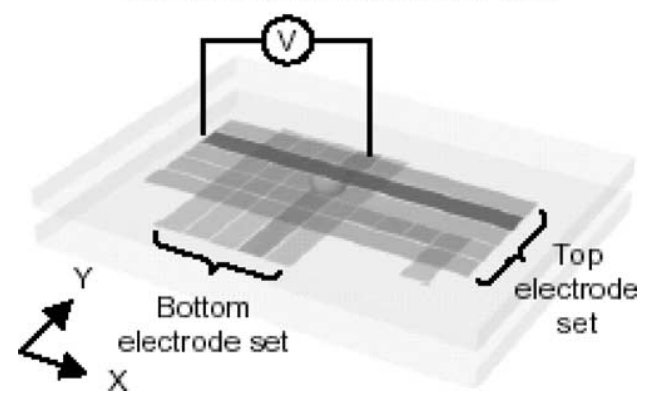

(a)
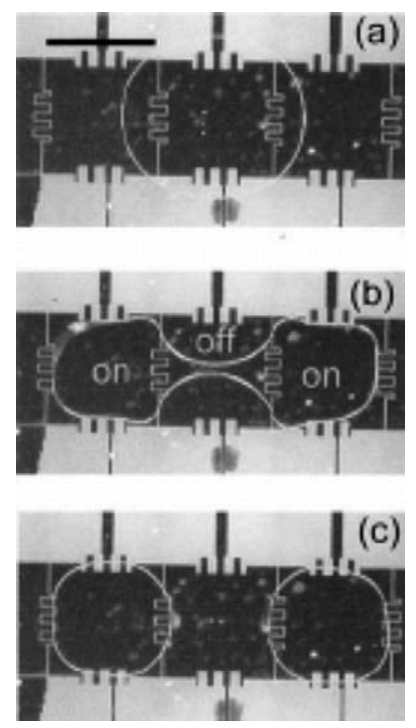

(b)
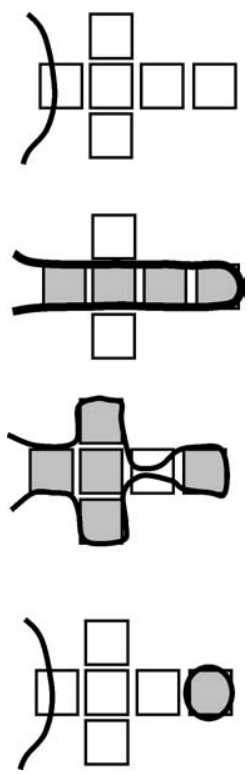

(c)

Fig. 3. Validation des fonctions de base : (a) déplacement dans un plan [22]; (b) morcellement [6]; (c) formation d'une goutte à partir d'un réservoir [6].

\section{Les composants discrets confinés}

Dans la littérature, les composants discrets confinés sont les plus souvent présentés. Ils sont généralement formés d'un assemblage de deux plaques parallèles et espacés de quelques dizaines à quelques centaines de microns. Les gouttes sont injectées dans cet entrefer et prises en sandwich. Généralement l'une des plaques comporte une matrice d'électrodes adjacentes de commande, l'autre plaque comporte une électrode de référence. Les plaques sont recouvertes d'une couche isolante et hydrophobe.

\subsection{Déplacement}

Grâce à un simple système de relais électrique, il est possible d'actionner électriquement les différentes électrodes, et donc de piloter les zones où l'électromouillage doit agir. Une goutte à cheval sur deux électrodes dont l'une d'elle est actionnée se déplace vers l'électrode activée. Des vitesses de déplacement impressionnantes ont étés observées $(250 \mathrm{~mm} / \mathrm{s})$ [6].

Dans le cas d'un composant dédié au haut débit d'analyse, un grand nombre de gouttes doivent être commandées via de grandes matrices de $N \times M$ électrodes ( $N$ et $M$ égaux à 8,16 voire 256). Le nombre $(N \times M)$ de connexions électriques impliquées devient important à moins d'utiliser une matrice active multiplexée. En superposant orthogonalement deux substrats [22] ayant chacun un réseau de lignes parallèles, la matrice résultante (Fig. 3(a)) comporte bien $N \times M$ électrodes pilotées avec seulement $N+M$ relais de commandes.

\subsection{Fusion et morcellement}

L'optimisation de la vitesse d'homogénéisation du mélange après fusion par coalescence a été étudiée par [23]. La fonction inverse, qui est énergiquement plus coûteuse est le morcellement d'une goutte. En effet la pression dans chaque goutte après fragmentation est plus importante que la pression initiale, ce qui a des conséquences sur le dimensionnement du composant [6]. Le principe consiste à étaler la goutte sur trois électrodes puis de couper l'activation sur l'électrode du milieu.

\subsection{Formation}

La dernière fonction rapportée sur la Fig. 3(c) permet d'engendrer des gouttes à partir d'un réservoir. Le principe est voisin de celui du morcellement. Une amélioration consiste à placer deux électrodes latérales ce qui permet d'après [6] de pomper le 
liquide pendant la formation du col et ainsi de garantir une meilleur reproductibilité du processus quel que soit le volume de liquide dans le réservoir.

Ainsi toutes les fonctions de base nécessaires à un protocole fluidique intégré ont été démontrées. Cependant cette première famille de configurations présente des inconvénients spécifiques : les deux substrats doivent être assemblée avec une contrainte de parallélisme pour éviter les pompages capillaires (coin d'huile) et le couvercle doit être connecté électriquement. De plus la présence du couvercle pour le confinement des gouttes rapproche les solutions techniques envisageables de celles qui sont utilisées pour des micro canaux. Les inconvénients qui en résultent sont donc les mêmes : la mise en oeuvre nécessite des ouvertures ou des puits; les gouttes fortement comprimées entre les plans ont un rapport surface sur volume du même ordre que les bouchons de la microfluidique continue; les risques d'absorption d'entités biologiques sur les parois sont importants, favorisant la contamination croisée entre gouttes, ou inversement, un déficit de concentration après un long déplacement.

\section{Les composants discrets ouverts}

Une autre famille de dispositifs, dans lesquels la goutte repose simplement sur un support actif, présente des avantages de simplicité et de coût. De plus, un système ouvert permet d'utiliser des méthodes de distribution classique comme par exemple une simple pipette ou un robot dispensateur. Enfin une goutte, gardant la forme d'une calotte sphérique, voire d'une sphère dans le cas d'une surface super-hydrophobe, présente le plus bas rapport possible de surface de contact liquide-solide sur volume de goutte.

\subsection{Déplacement et injection}

Pour le déplacement, différents principes ont été proposés. Il a été montré que l'on pouvait déplacer une goutte par champ électrostatique progressif émis par des peignes d'électrodes interdigitées [24]. Pour décrire le déplacement, il faut à la fois tenir compte de l'électromouillage mais aussi des contraintes électriques normales qui figurent dans le membre de droite de l'Éq. (1). La formation d'une goutte étudiée par Jones [25], consiste à mettre en oeuvre les contraintes électriques évoquées ci-dessus à travers des fluides diélectriques; pour cela le liquide de la goutte doit être faiblement salin et les charges libres sont de plus immobilisées par une fréquence très élevée. La résultante, dite force diélectrophorétique, a pour effet de pousser le liquide le plus diélectrique vers les zones de champ fort. Ceci permet de confiner ou d'étaler un liquide entre deux lignes électriques.

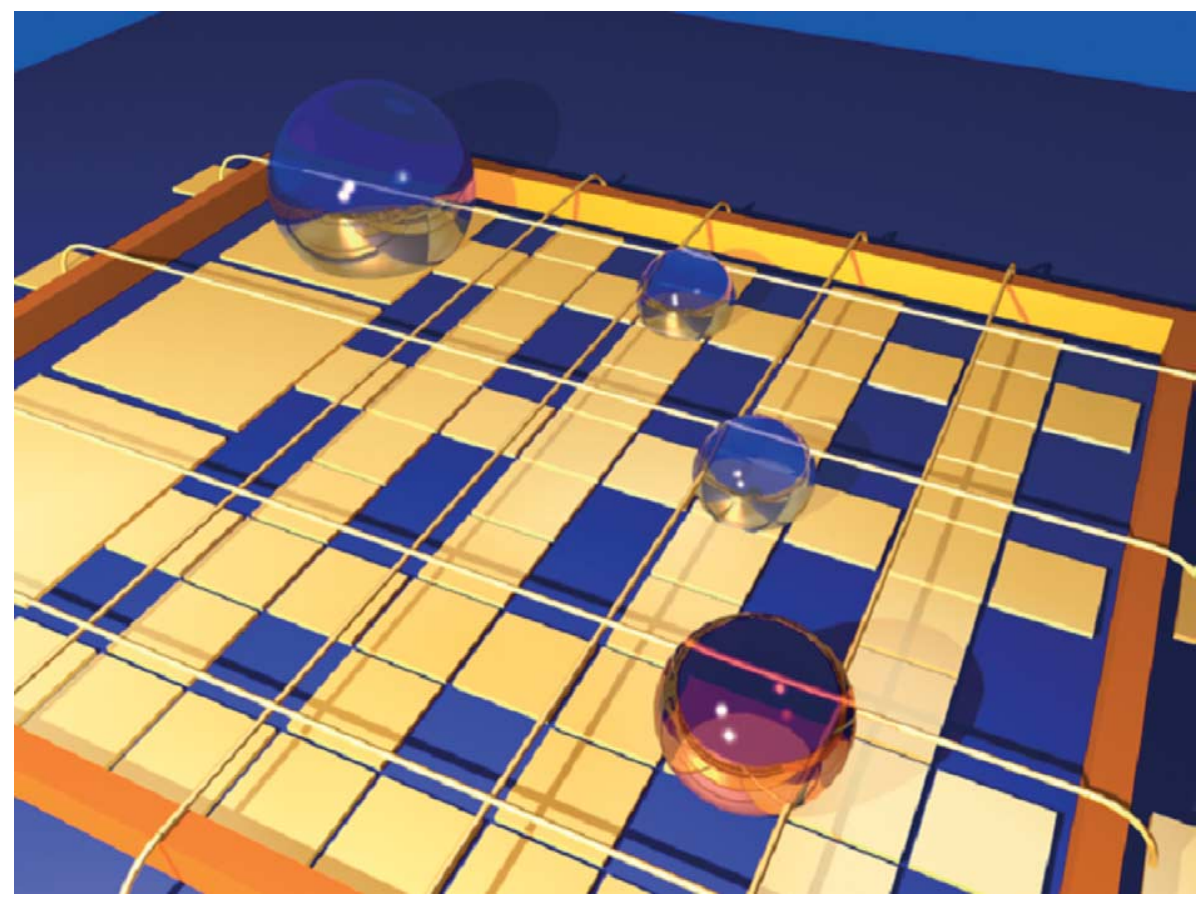

Fig. 4. Exemple de dispositif ouvert mettant en évidence le réseau de micro-caténaire (image de synthèse). 

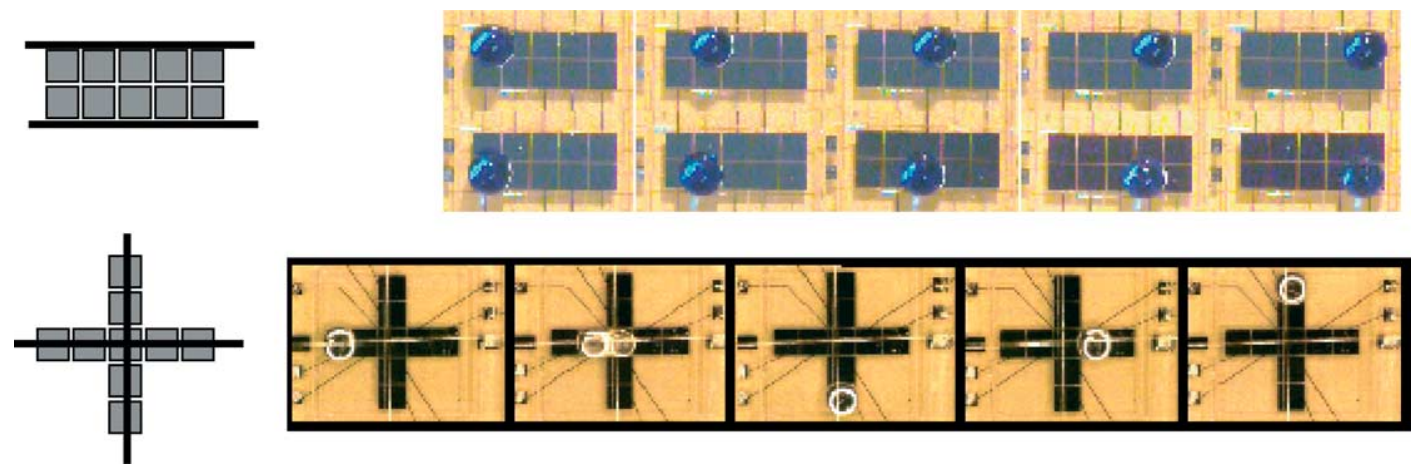

Fig. 5. Manipulation par électromouillage de gouttes sur un plan dans un système ouvert. Boucles (5-1) et aiguillage (5-2). (100 volts et 3 KHz.)

Des déplacements ont également été obtenus à partir de différents composants utilisant l'électromouillage sur isolant, tout en gardant la possibilité d'avoir un système ouvert [26]. Pour cela, un micro fil électrique est tendu au dessus d'une surface comportant des électrodes isolées (Fig. 4). Le caténaire a un double rôle de guidage et d'électrode : la goutte se déplace à la fois comme un téléphérique et un tramway. Le caténaire est réalisée, sans qu'une grande précision de positionnement soit exigée, lors de l'implantation des connexions électrique de la puce. En revanche il faut être attentif au mode de mouillage de la goutte sur le caténaire. Dans le cas d'une goutte d'eau pure [27], le caténaire reste tangent à la goutte (mouillage en perle) alors qu' avec un liquide contenant des tensio-actifs, le caténaire peut traverser la goutte (mouillage en tonneau).

La surface de mouillage d'une goutte de volume $\mathcal{V}$ en mouillage partiel est proportionnelle à $\mathcal{V}^{1 / 3}$, à comparer à la valeur $\mathcal{V}^{1 / 2}$ dans le cas d'un système confiné. Par conséquent, une même configuration permet d'utiliser des électrodes plus petites et de déplacer des gouttes d'une gamme plus étendue de volumes. Pour fixer les idées, avec des électrodes de $800 \mu \mathrm{m}$ de coté, des gouttes de $0.5 \mu \mathrm{l}$ à plus de $2 \mu \mathrm{l}$ peuvent être déplacées.

La fonction boucle (Fig. 5-1) permet à une goutte de quitter un caténaire pour sauter sur une autre piste. De même une structure en croix permet à une goutte de changer de direction (Fig. 5-2). Ainsi, on remarque que la force de mouillage des gouttes sur le caténaire reste faible devant les forces engendrées par l'électromouillage. Par ailleurs, le multiplexage de la commande est naturellement applicable ici en superposant orthogonalement les caténaires à des lignes d'électrodes.

Enfin notons que l'on est conduit à envisager simultanément l'implantation des connexions électriques et fluidiques sur la base d'un même composant. Pour les applications souhaitées, il en résulte une grande flexibilité.

\subsection{Fusion dans l'air et mélange}

Le mélange par coalescence entre deux gouttes s'effectue simplement à la suite du microécoulement résiduel dans la goutte finale qui est induit par le processus même de fusion et qui n'est pas (ou peu) ralenti par des frottements pariétaux. De même, l'homogénéisation du mélange dans une goutte $1.7 \mu \mathrm{l}$ ne demande plus quelques minutes mais quelques secondes par la simple mise en mouvement de la goutte sur quelques électrodes [28].

\subsection{Fusion dans un liquide visqueux}

Suivant le début de ce chapitre, un système ouvert permet d'utiliser des méthodes de distribution classique; dans le cas où ces dernières aboutissent à une fusion de deux gouttes, une goutte posée déjà insérée dans le système et une goutte de réactif par exemple venant de l'extérieur, l'expérience montre que l'occurrence de la dite fusion est aléatoire, notamment dans le cas où le fluide entourant la goutte est visqueux. Pour assurer un drainage efficace du fluide interstitiel, on impose pendant un court instant, un champ électrique suivant l'axe passant par le centre des gouttes : il s'agit d'un processus d'électro-coalescence dont les trois principales étapes sont présentées Fig. 6.

Si la distance entre les gouttes est supérieure au diamètre de la goutte pendante, cette dernière se détache alors de l'injecteur avant de fusionner avec la goutte posée (Fig. 7(a)); il n'y a pas de contact physique entre goutte inférieure et injecteur, et donc aucune contamination de l'injecteur par le liquide de la goutte fixe.

\subsection{Mélange}

Dans le cas précédent, l'union des deux masses fluides se produit par encapsulation de la goutte émise par la goutte réceptrice. A la fin de ce phénomène, une structure en forme de «champignon» apparaît (Fig. 7(b)), décrite par [29]. 


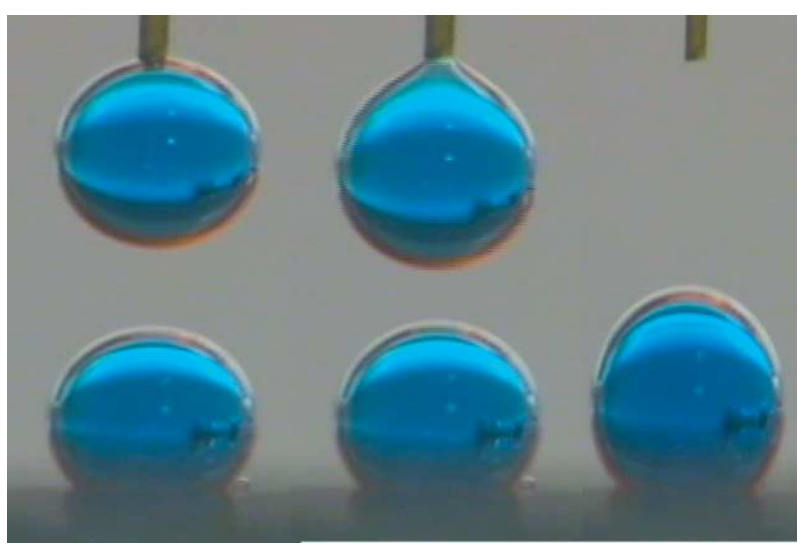

Fig. 6. Les principales étapes, décrites de gauche à droite, d'une expérience de coalescence : (a) Etat initial, aucun champ n'est imposé. Les deux gouttes sont sphériques; (b) On impose une différence de potentiel (durée : $50 \mathrm{~ms}$ ) au système et les deux gouttes se déforment; (c) La goutte pendante s'est détachée de l'aiguille (ddp : 540 volts) et a fusionné avec la goutte posée.

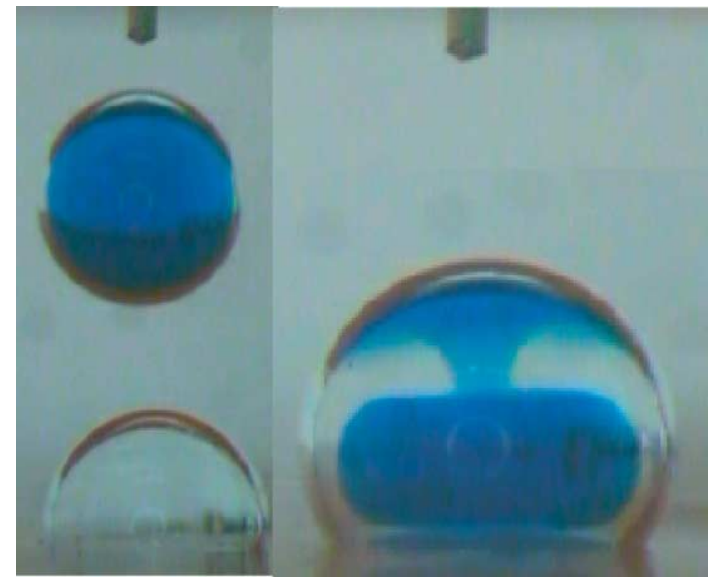

Fig. 7. Les principales étapes, décrites de gauche à droite, de l'encapsulation : (a) goutte «volante»; (b) état initial du mélange : la structure «champignon».

C'est la diffusion naturelle qui assure le mélange uniforme, mais lent, des deux liquides en une dizaine de secondes. On peut accélérer ce mélange en soumettant la goutte à un champ électrique continu. Une injection d'ions par effet de pointe se produit au niveau de l'électrode, ici un capillaire [30]. L'huile se met en mouvement autour de la goutte et induit un brassage à l'intérieur de celle-ci.

\section{Conclusions}

Dans les Sciences du Vivant, une partie de la microfluidique (ou de la nanofluidique) vise à apporter des solutions instrumentales pour observer ou manipuler des objets biologiques individuels et ainsi améliorer l'étude de certains mécanismes fondamentaux. De fait, la microfluidique a majoritairement partie liée aux bio-technologies qui poursuivent deux grandes classes d'objectifs. Un premier axe se développe en relation avec la recherche pharmaceutique. L'identification des gènes tout d'abord, qui devait donner naissance à une nouvelle génération de molécules personnalisées, plus ciblées et plus efficaces. Après des réussites mitigées, cet axe de recherche se poursuit aujourd'hui par le recensement des protéines codées par ces gènes et puis par l'identification des messagers chimiques entre cellules. Ce type de recherche requiert la mise au point de microsystèmes de type haut débit d'analyse. Le second axe se développe en relation avec des milieux socio-professionnels variés : médical, agro-alimentaire, environnement. . . Il s'agit du diagnostic, avec la notion du labopuce. Les objectifs poursuivis dans le cadre de ces deux axes sont très proches et comportent des étapes communes : préparation des échantillons, séparation et analyse. Ces 
étapes doivent être réalisées automatiquement dans des microsystèmes. La réalisation de micro-systèmes, suffisamment fiables pour être industrialisés, s'inscrit dans la constitution d'un nouveau génie des procédés, adapté aux petites échelles d'une part et permettant d'autre part d'intégrer de nombreuses opérations relatives à un processus donné. Dans ce génie des procédés, la microfluidique apparaît comme la discipline scientifique maîtresse, qui se situe au carrefour de nombreuses autres disciplines.

Ainsi toute l'ingénierie des systèmes fluidiques à grande échelle a du être repensée. La «microfluidique continue» a été la première réponse à ce défi. Elle reste actuellement l'approche dominante et il est juste de dire qu'elle compte des réussites incontestables. Toutefois, nous en avons délibérément souligné les faiblesses spécifiques ; il nous paraît important de s'affranchir de ce qui nous paraît être ses inhérentes impasses en défrichant d'autres pistes : la «microfluidique dicrète » est une de ces pistes. Son couplage avec les microtechnologies, dérivée de l'électronique, apparaît encore plus naturel et rend cette microfluidique particulièrement attractive. Il s'agissait tout d'abord de valider les principales fonctions fluidiques de base mais surtout de montrer ensuite que l'intégration de ces fonctions au sein de systèmes complexes (mise en série ou en parallèle) pouvait être plus aisée. Pour conclure temporairement sur cette comparaison, on peut imaginer que les systèmes de demain réuniront dans une approche hybride les solutions les plus avantageuses issues des deux microfluidiques.

Il reste cependant un grand nombre d'études à mener pour concrétiser ces promesses. Les études fondamentales doivent s'attacher prioritairement aux couplages subtils entre l'électroquasistatique et la mécanique des fluides des interfaces fluide/fluide ou fluide/solide; le but est d'affiner ou de généraliser les modèles physiques existant à des situations plus complexes, mais plus proches de la réalité, propres à la microfluidique discrète. Ensuite, il convient de s'assurer que les modèles physiques utilisés restent encore valides pour des liquides biologiques. Cela passe évidemment par une meilleure compréhension des phénomènes physico-chimiques aux interfaces. Malgré son aptitude à assurer les fonctions fluidiques de base dans une combinatoire parfaitement maîtrisée, la microfluidique discrète ne pourra convaincre dans les années à venir que si elle à arrive à traiter l'ensemble de protocoles chimiques ou biologiques complexes. En effet, un résultat d'analyse ne se ramène pas toujours à une simple mesure de changement de couleur. L'enjeu est véritablement de descendre d'un niveau la commande des processus : la goutte, microréacteur contrôlable dans sa globalité, doit également l'être au niveau des réactions qui se produisent en son sein. On retrouve ainsi pour ce microréacteur, le caractère pluridisciplinaire habituel de la microfluidique : il s'agit d'exploiter intelligemment certains phénomènes mécaniques, chimiques, électriques [31], ou thermiques [32] qui, aux micro-échelles où ils sont observés, se trouvent généralement fortement couplés.

\section{Références}

[1] S.C. Terry, Ph.D. Dissertation, Stanford University, 1975.

[2] A. Manz, N. Graber, H.M. Widmer, Sensors and Actuators B 1 (1990) 244-248.

[3] R.A. Mathies, P.C. Simpson, A.T. Woolley, in: Proc. of Micro Total Analysis Systems, 1998, pp. 1-6.

[4] T. Torsen, S.J. Maerki, S.R. Quake, Science 298 (2002) 580, 584.

[5] D. Reyes, D. Lossifidis, P.A. Auroux, A. Manz, Anal. Chem. 74 (2002) 2623-2636.

[6] S. Kwon Cho, H. Moon, C.J. Kim, J. Microelectromech. Systems 12 (1) (2003).

[7] A.L. Yarin, W. Liu, D.H. Reneker, J. Appl. Phys. 91 (7) (2002) 4751, 4760.

[8] A. Buguin, L. Talini, P. Silberzan, Appl. Phys. A 75 (2002) 207-212.

[9] A. Castellanos, Electrohydrodynamics, Springer, Wien, 1998.

[10] A.J. Tudos, G.A.J. Besselink, B.M. Schasfoort, Lab on a Chip 1 (2001) 83-95.

[11] Y.K. Lee, J. Deval, P. Tabeling, C.M. Ho, Proc. IMRET 5 (2001).

[12] W.N. Gill, R. Sankarasubramanian, Proc. Roy. Soc. London Ser. A 316 (1970) 341-350.

[13] J. Sudor, Y. Fouillet, N. Sarrut, I. Chartier, P. Peltie, A. Gruss, in : Publications S.H.F., Congrès Microfluidique, Toulouse, 2002, pp. 291297.

[14] J. Darabi, M. Rada, M. Ohadi, J. Lawker, J. Microelectromech. Systems 11 (6) (2002) 684.

[15] J.C. Slattery, Interfacial Transport Phenomena, Springer-Verlag, 1990.

[16] T.B. Jones, Electromechanics of Particles, Cambridge University Press, 1995.

[17] G.I. Taylor, Proc. Roy. Soc. A 280 (1964) 383.

[18] B. Berge, C.R. Acad. Sci. Paris, Sér. II 317 (1993) 157-163.

[19] F. Saeki, J. Baum, H. Moon, J.Y. Yoon, C.J. Kim, R.L. Garrell, Polym. Mater. Sci. Engrg. 85 (2001) 12-13.

[20] F. Mugele, S. Herminghaus, Appl. Phys. Lett. 81 (12) (2002) 2303-2305.

[21] K.H. Kang, I.S. Kang, C.M. Lee, Langmuir 19 (2003) 5407-5412.

[22] S.K. Fan, P.P. de Guzman, C.J. Kim, in: Solid State Sensor, Actuator and Microsystems Workshop South Carolina, June 2-6, 2002, pp. 134-137.

[23] P. Paik, V.K. Pamula, M.G. Pollack, R.B. Fair, Lab on a Chip 3 (2003) 28-33.

[24] M. Washizu, in: IEEE Industry Applications Society. Annual Meeting, New Orleans, LA, October 5-9, 1997, pp. 1867-1873.

[25] T.B. Jones, J. Electrostatic 51-52 (2001) 290-299.

[26] Y. Fouillet, H. Jeanson, I. Chartier, A. Buguin, P. Siliberzan, La Houille blanche 4 (2003) 37-42.

[27] G. McHale, N.I. Newton, B.J. Carroll, Rev. IFP 56 (1) (2001) 47-54. 
[28] Y. Fouillet, H. Jeanson, D. Jary, O. Constantin, in: Proceeding of Micro Total Analysis Systems, 2003.

[29] O. Fuchs, Y. Fouillet, J.-L. Achard, in: Publications S.H.F., Congrès Microfluidique, Toulouse, 2002, pp. $37-46$.

[30] P. Atten, D2850 Techniques de l'Ingénieur, traité génie électrique, 1996.

[31] S.K. Cho, C.J. Kim, in: IEEE Conf. MEMS, Kyoto, Japan, January 2003, pp. 686-689.

[32] P. Pham, J.-L. Achard, J. Berthier, F. Ginot, in: Nanotechnology Conference and Trade Show, vol. 1, San Francisco, 23-27 February, 2003, pp. 202-205. 